\title{
Controlling and manipulation of red blood cells by evanescent waves
}

\author{
O.V. ANGELSKY ${ }^{1,2^{*}}$, C.YU. ZENKOVA ${ }^{1}$, P.P. MAKSYMYAK ${ }^{1}$, \\ A.P. MAKSYMYAK ${ }^{1}$, D.I. IVANSKYI ${ }^{1}$ \\ ${ }^{1}$ Chernivtsi National University, Kotsyubynsky 2, 58012 Chernivtsi, Ukraine \\ ${ }^{2}$ Taizhou Research Institute of Zhejiang University, China \\ ${ }^{*}$ Corresponding author: o.angelsky@chnu.edu.ua
}

\begin{abstract}
A theoretical model for describing the influence of the evanescent wave on the red blood cell (RBC, erythrocyte) in the blood plasma is suggested in this research work. Two optical schemes for creating an evanescent wave and the features of the created field's effect on the erythrocyte are considered. The conditions for the formation of optical forces and optical momentum, in particular, of the vertical spin of the evanescent wave, which causes a transverse displacement of the erythrocyte, are proposed. The use of a linearly polarized plane wave with azimuth of $\pm 45^{\circ}$ in a model experiment, specially suggested in this work, allows for visualization of the transverse controlled motion of the erythrocyte, which enables to claim about new possibilities for controlling microobjects in biology and medicine.
\end{abstract}

Keywords: erythrocytes, evanescent wave, spin momentum.

\section{Introduction}

A majority of investigations in biology and medicine carried out for the last decades are devoted to the development of methods for studying biological objects with visualization of micromanipulations at molecular and cell scales. Noninvasive optical trapping and moving the microobjects presume application of the approaches characterized by superhigh accuracy [1] and used in investigations on biological molecules [2- $\underline{6}$ ], red blood cells, etc. As a rule, controlled manipulations of microobjects are performed using optical tweezers. At present, a set of techniques is available for manipulation of biological objects and microparticles using physical nature tweezers, by transfer of the linear momentum from a photon. Transfer of the linear momentum from light to particles depends on the optical properties of the controlled microparticles and on the phase distribution [ $[-10]$ and polarization [11-13] of the probing beam.

A new method for controlling a biological microobject, in particular, the one with an erythrocyte in the especially selected optical scheme, by forming an evanescent wave 
with a pronounced transverse component of the spin moment, is suggested for consideration in this research.

Evanescent waves excited above the interface of two media with different refraction indices are the complementary source of optical forces and optical torques, which can be employed for the manipulation of microparticles in a broader range of applications as compared with conventional tweezers.

It is of importance to search for techniques for manipulation of biological microobjects at the cell scale in vivo, by use of the physiological permeability of biomembranes. The use of optical tweezers for trapping and manipulating erythrocytes in a blood capillary gives a possibility to clean microvessels and renew their permeability owing to the combination of the results of biological investigations in vitro and in vivo [14]. The ability of laser radiation to penetrate biological tissues enables a creation of systems for noninvasive manipulation of biological micro- and nanoobjects in living organisms, including realization of controlled spatial motion of such objects, while avoiding the tissue destruction.

The progress in diagnostic and therapeutic techniques used in practical medicine promotes further search for tools for controlled movement of nanoobjects, including RBCs in biological systems.

Thus, the purpose of this work is to search for new approaches to control the evanescent wave by spatial location of an isolated RBCs in a blood plasma, with an experimental demonstration of the predicted motion and corresponding selection of the irradiation conditions, with preceding matching of the experimental parameters to a theoretical model, for calculating the evanescent wave in the erythrocyte.

\section{Calculation algorithm of erythrocyte interaction with an evanescent wave}

Meanwhile, in the case of isolated erythrocytes, the question about the feasibilities for controlled motion of these cells using an evanescent wave excited at the boundary of "prism-blood plasma" remains unsolved. To enhance the feasibilities for noninvasive control of RBCs using laser radiation, we propose to excite an evanescent wave using a linearly polarized wave with an azimuth of polarization $\pm 45^{\circ}$, giving transversal spin flows in the evanescent wave and controlled spatial (transversal) motion of erythrocytes.

The normal human erythrocyte has the shape of concavo-concave disc $[\underline{15}, \underline{16}]$. The diameter $D$ of an erythrocyte is $7.82 \pm 0.62 \mu \mathrm{m}$, and its minimal $b$ and maximal $h$ thickness at the centre and at the limb are $0.81 \pm 0.35 \mu \mathrm{m}$ and $2.58 \pm 0.27 \mu \mathrm{m}$, respectively. The mean magnitude of the cell volume of an erythrocyte is $94 \pm 14 \mu \mathrm{m}^{3}$, and the mean magnitude of the cell surface area is $135 \pm 16 \mu \mathrm{m}^{2}$ [15]. In our case, $D=7.2 \mu \mathrm{m}$, $b=0.9 \mu \mathrm{m}, h=2.4 \mu \mathrm{m}$, and the distance between two maximal central points in the transversal cross-section $c=4.74 \mu \mathrm{m}[17]$. RBCs are deformed in a shearing flow, where their shape becomes ellipsoidal [18-20]. Considering an erythrocyte as a uniform disc with the mean erythrocyte volume, one can use the concept of single light-scattering 
by a cell where the cell organelles are believed to be small enough and the cell membrane thin enough to neglect their influence on light scattering properties. The Mie theory of light scattering applied for such cases provides a comprehensive description of the energy transfer to the erythrocyte [15].

Modelling of a RBC by a disc facilitates the determination of an algorithm that represents a cell in an evanescent field. We are interested in understanding the transversal motion of such a cell. This becomes possible if the probing wave is linearly polarized, with the azimuth of polarization $\pm 45^{\circ}$. An elliptically polarized evanescent incident wave introduces a vertical spin angular momentum. This introduces transversal and longitudinal forces, inducing motion of the erythrocyte. The formed evanescent wave, being elliptically polarized, has a torque $\tau$ causing rotation of an erythrocyte.

In contrast to previous studies of induced longitudinal motion induced by an evanescent field, we will here study transversal motion. This motion is introduced by a combination of the incident field and its polarization.

Observation of motion of an isolated RBC in the whole blood is hampered by high concentration of cells. Concentration of erythrocytes is diminished using an isotonic solution. However, the simplest use of physiological solution is not practical due to the lower viscosity of the solution changing the elasticity of erythrocytes. Consequently, we diminished the RBC concentration using the natural medium of cells, viz. the blood plasma. Following this approach, both computer simulation and experimental demonstration of erythrocyte motion are carried out in the blood plasma with refractive index $\left(n_{\text {sol }}=1.37\right)$. To realize the total internal reflection, we use a flint prism with an index of refraction $n_{\mathrm{pr}}=1.61$, above which an erythrocyte with the refraction index $n_{\mathrm{er}}=1.407$ is located [15] in the blood plasma. The entered refractive indexes correspond to the wavelength of $633 \mathrm{~nm}$. To avoid the temperature effects and irreversible temperature changes due to heating and destroying cells under illumination, one sets the power of radiation below $100 \mathrm{~mW}$ and the pulse cycling of about $1 \mu \mathrm{s}$.

For observation of the controlled transversal motion of an erythrocyte in the YOZ-plane (which is possible only if the transversal components of the evanescent wave are active), we propose to implement the model arrangement shown in Fig. 1, where two oppositely directed evanescent waves of the same amplitude and frequency are formed above the prism. In the model experiment, the longitudinal components of the optical force of the evanescent waves are compensated on the RBC, so that only the transversal components are added. Let us emphasize, the discussed effect only becomes possible if one uses two linearly polarized incident waves with the azimuths of polarization $\pm 45^{\circ}$ with respect to the plane of incidence, so that two oppositely directed evanescent waves are of the same elliptical polarization.

To simulate the interaction of the evanescent wave with the erythrocyte as a complex-shaped object, we break the surface of the erythrocyte into a set of facets of equal areas of $0.01 \mu^{2}$. The chosen size of the facets is necessary and sufficient for the description of the erythrocyte surface interacting with an evanescent wave. With respect to the transversal size $h$ of an erythrocyte, $h / 3$ takes part in the interaction of the cell and the evanescent wave, which was obtained due to preliminary calculations. The sug- 


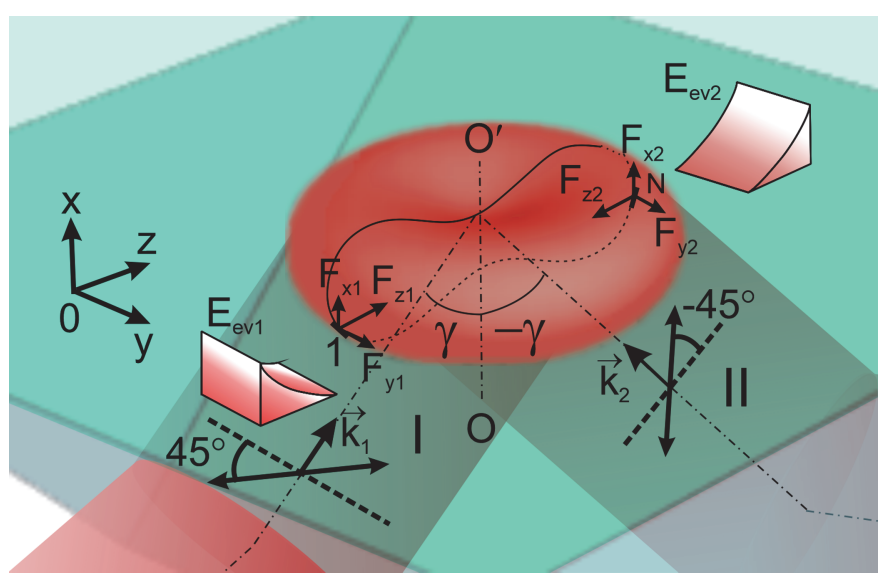

Fig. 1. Two linearly polarized beams I and II with azimuths of polarization $\pm 45^{\circ}$ with respect to the plane of incidence are incident on the prism at the angle $\gamma$. Here $\mathbf{k}_{1}$ and $\mathbf{k}_{2}$ are the wavevectors of the incident beams; $F_{x 1}, F_{x 2}, F_{y 1}, F_{y 2}, F_{z 1}, F_{z 2}$ are the components of the optical force of the evanescent waves $E_{\text {ev1 }}$ and $E_{\text {ev } 2} ; O O^{\prime}$ is the axis of symmetry of an erythrocyte; $N=1 \ldots i$ - the number of simulating facets.

gested modelling will enable to estimate the components of the optical force $F_{x}, F_{y}$, and $F_{z}$ for each facet and the resulting components in the $x, y$, and $z$ directions. As a consequence, it becomes possible to determine the transverse optical force.

Computer simulations were carried out for 3340 facets interacting with an evanescent wave adding up the derived components of the optical force for three directions for each facet.

In doing so, one takes into account the angle of incidence of radiation at the corresponding facet and optical characteristics of the erythrocyte surface. During the simulation, the coordinates are obtained from the following equation describing the shape of a RBC as a concavo-concave disc [17]:

$$
\left(y^{2}+z^{2}\right)^{2}+2 S\left(y^{2}+z^{2}\right) x^{2}+x^{4}+P\left(y^{2}+z^{2}\right)+Q x^{2}+R=0
$$

Correspondingly, the parameters $P, Q, R$, and $S$ can be determined as:

$$
\begin{aligned}
& P=-\frac{D^{2}}{4}-\frac{b^{2} h^{2}}{4 D^{2}}+\frac{b^{2} c^{4}}{4 D^{2}\left(h^{2}-b^{2}\right)} \\
& Q=\frac{D^{4}+4 D^{2} P-b^{4}}{4 b^{2}} \\
& R=-\frac{D^{4}}{16}\left(D^{2}+4 P\right) \\
& S=-\frac{c^{2}+2 P}{h^{2}}
\end{aligned}
$$




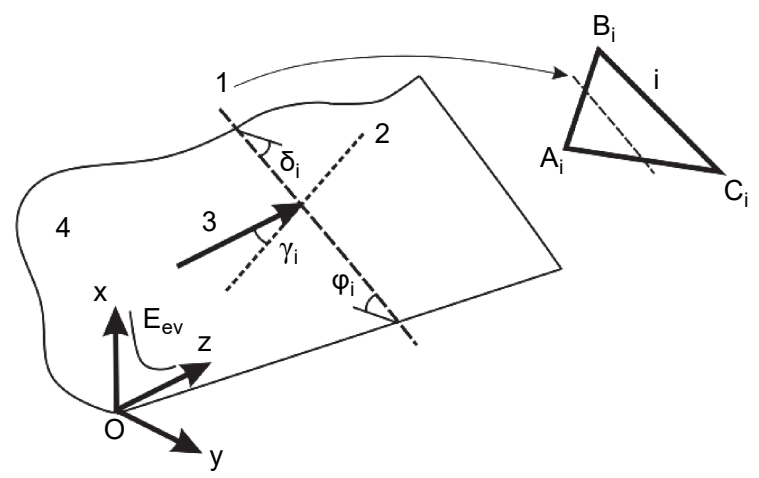

Fig. 2. Model describing the incidence of an evanescent wave $E_{\mathrm{ev}}$ at the $i$-th facet $\left(A_{i} B_{i} C_{i}\right)$ of the erythrocyte surface: $X Y Z$ are the coordinates determined by the condition of formation of an evanescent wave, corresponding to Fig. $1 ; 1$ - the intersection line of incidence plane with the $i$-th facet $\left(A_{i} B_{i} C_{i}\right) ; 2$-normal at the point of incidence of the beam 3 (which coincides with the direction of $\mathbf{b}$ ) at the facet. The angle of incidence of the beam at the facet $\gamma_{i}$ determines the plane of incidence 4 making angle $\varphi_{i}$ with the $Y O Z$-plane and an angle $\delta_{i}$ with the $X O Y$-plane.

Let us set the coordinates $X Y Z$ with the origin at point $O(0,0,0)$, as it is shown in Fig. 2. The coordinates of facets are measured from this point to the right and to the left along the axes $O Y$ and $O Z$ with the stepsize $0.01 \mu \mathrm{m}$. This stepsize provides the optimized condition for computer simulation. Following the above equations for each $(y, z)$ position, one determines the magnitude $x$ for the point at the erythrocyte surface. The areas of the facets chosen for simulation $\left(\sim 0.01 \mu \mathrm{m}^{2}\right)$ are determined using computer simulation. The upper magnitude of the coordinate along the $X$-axis is chosen in correspondence with the preliminary estimated depth of penetration of an evanescent wave into the RBC. The use of software Mathematica enables such division in triangular facets of equal areas, with determination of the apex of each facet, which is used for simulation of the erythrocyte surface. Using the mentioned software, one can estimate the coordinates of the each point of a facet, as well.

The obtained set of facets is distinct in its spatial position in the specified coordinates $X Y Z$ (Fig. 2).

Let us arbitrarily consider the $i$-th facet whose apexes are determined as $A_{i}$ $\left(x_{A_{i}}, y_{A_{i}}, z_{A_{i}}\right), B_{i}\left(x_{B_{i}}, y_{B_{i}}, z_{B_{i}}\right)$, and $C_{i}\left(x_{C_{i}}, y_{C_{i}}, z_{C_{i}}\right)$. Then, the plane containing this facet can be determined as $a_{i} x+b_{i} y+c_{i} z+d_{i}=0$, where the coefficients $a_{i}, b_{i}, c_{i}, d_{i}$ are:

$a_{i}=\left(y_{A_{i}}-y_{C_{i}}\right)\left(z_{B_{i}}-z_{A_{i}}\right)+\left(y_{B_{i}}-y_{A_{i}}\right)\left(z_{C_{i}}-z_{A_{i}}\right)$

$b_{i}=\left(x_{C_{i}}-x_{A_{i}}\right)\left(z_{B_{i}}-z_{A_{i}}\right)+\left(x_{A_{i}}-x_{B_{i}}\right)\left(z_{C_{i}}-z_{A_{i}}\right)$

$c_{i}=\left(x_{A_{i}}-x_{B_{i}}\right)\left(y_{A_{i}}-y_{C_{i}}\right)+\left(x_{C_{i}}-x_{A_{i}}\right)\left(y_{A_{i}}-y_{B_{i}}\right)$

$d_{i}=\left(x_{C_{i}}-x_{A_{i}}\right)\left(y_{B_{i}} z_{A_{i}}-y_{A_{i}} z_{B_{i}}\right)+\left(y_{A_{i}}-y_{C_{i}}\right)\left(x_{B_{i}} z_{A_{i}}-x_{A_{i}} z_{B_{i}}\right)+\left(z_{C_{i}}-z_{A_{i}}\right)\left(x_{B_{i}} y_{A_{i}}-x_{A_{i}} y_{B_{i}}\right)$

Here, the normal vector for the specified facet is determined by $\mathbf{n}_{i}\left(a_{i}, b_{i}, c_{i}\right)$. 
An evanescent wave propagating along the $O Z$-direction reaches the specified $i$-th facet and makes some angle $\gamma_{i}$ with the normal to this facet at the point of incidence of the beam (Fig. 2). If the vector determining the direction of propagation of the incident beam is determined as $\mathbf{b}\left(0,0, c_{i}\right)$, then

$$
\cos \gamma_{i}=\frac{\left|c_{i}\right|}{\sqrt{a_{i}^{2}+b_{i}^{2}+c_{i}^{2}}}
$$

Three coordinates for the specified facet determine the point of incidence of a beam onto the facet, being arbitrarily chosen. Consequently, the normal and the incident beam determine the plane of incidence, and one can use the Fresnel equations to compute the components of the electrical field of the reflected and refracted waves, and to determine the angle of incidence $\gamma_{i}$ between the beam and the surface normal. Correspondingly, one can estimate the refraction angles $\gamma_{i}^{\mathrm{t}}$ for the incident beam at each facet. The orientation of the plane of incidence with respect to the horizontal YOZ-plane, i.e. the angle $\varphi_{i}$, can be estimated by

$$
\cos \varphi_{i}=\frac{\left|b_{i} c_{i}\right|}{\sqrt{a_{i}^{2} c_{i}^{2}+b_{i}^{2} c_{i}^{2}}}
$$

calculated during the computer simulation. Correspondingly, one can determine the orientation of the plane of incidence with respect to the vertical XOZ-plane, i.e. the angle $\delta_{i}$, giving

$$
\cos \delta_{i}=\frac{\left|a_{i} c_{i}\right|}{\sqrt{a_{i}^{2} c_{i}^{2}+b_{i}^{2} c_{i}^{2}}}
$$

\subsection{The computation of the optical force generated by one evanescent wave}

An evanescent wave, being excited above the interface, can be described by the following equation [21]:

$$
\begin{aligned}
\mathbf{E}_{\mathrm{ev}}= & E\left(\mathbf{x} \frac{1}{\sqrt{1+|m|^{2}}}+\mathbf{y} \frac{m}{\sqrt{1+|m|^{2}}} \frac{k}{k_{z}}+\mathbf{z}(-i) \frac{1}{\sqrt{1+|m|^{2}}} \frac{\kappa}{k_{z}}\right) \\
& \times \exp (-\kappa x) \exp \left[i\left(k_{z} z-\omega t\right)\right]
\end{aligned}
$$

Here $k_{z}$ is the propagation constant, $\kappa$ is the exponential decay rate, $m$ is the state of polarization of an evanescent wave [22, 23] , and:

$$
k_{z}=k \frac{n_{\mathrm{pr}}}{n_{\mathrm{sol}}} \sin \gamma
$$




$$
\begin{aligned}
& \kappa=k \sqrt{\left(\frac{n_{\mathrm{pr}}}{n_{\mathrm{sol}}}\right)^{2} \sin ^{2} \gamma-1} \\
& m=\frac{T_{\perp}}{T_{\|}} m_{1}
\end{aligned}
$$

where $m_{1}$ is the state of polarization of the probing beam impinging on the interface "prism-blood plasma", being equal to unity for linear polarization with the azimuth of polarization $45^{\circ}$, and $\gamma$ is the incidence angle on the surface, where total internal refection takes place, $k$ is the wave number for the incident wave. The electrical field strength of an evanescent wave is

$$
E=\frac{k_{z}}{k} \sqrt{\frac{\mu_{1}}{\mu}} T E_{0}
$$

where $T$ is the transmission coefficient [24], and

$$
T=\frac{\sqrt{\left|T_{\|}\right|^{2}+\left|m_{1}\right|^{2}\left|T_{\perp}\right|^{2}}}{\sqrt{1+\left|m_{1}\right|^{2}}} \exp \left(i \arg T_{\|}\right)
$$

and $T_{\|}, T_{\perp}$ are the Fresnel transmission coefficients, $E_{0}$ and $\omega$ are the strength and frequency of the electrical field of the incident wave, respectively.

At the coordinates $X Y Z$, for the plane of incidence, one can write the components of the electrical vector of the reflected and refracted waves following the Fresnel equations. Therefore, for the reflected wave and the $i$-th facet one has:

$$
\begin{aligned}
& {\left[E_{\mathrm{ev} x}^{\mathrm{r}}\right]_{i}=\left[r_{\|}\right]_{i}\left[E_{\mathrm{ev} \|}\right]_{i} \sin \gamma_{i} \sin \varphi_{i}} \\
& {\left[E_{\mathrm{ev} y}^{\mathrm{r}}\right]_{i}=\left[r_{\perp}\right]_{i}\left[E_{\mathrm{ev} \perp}\right]_{i} \cos \varphi_{i} \sin \delta_{i}} \\
& {\left[E_{\mathrm{ev} z}^{\mathrm{r}}\right]_{i}=-\left[r_{\|}\right]_{i}\left[E_{\mathrm{ev} \|}\right]_{i} \cos \gamma_{i} \cos \varphi_{i} \cos \delta_{i}}
\end{aligned}
$$

Here

$$
\left\{\begin{array}{l}
{\left[r_{\|}\right]_{i}=\frac{n_{\mathrm{er}} \cos \gamma_{i}-n_{\mathrm{sol}} \cos \gamma_{i}^{\mathrm{t}}}{n_{\mathrm{er}} \cos \gamma_{i}+n_{\mathrm{sol}} \cos \gamma_{i}^{\mathrm{t}}}} \\
{\left[r_{\perp}\right]_{i}=\frac{n_{\mathrm{sol}} \cos \gamma_{i}-n_{\mathrm{er}} \cos \gamma_{i}^{\mathrm{t}}}{n_{\mathrm{sol}} \cos \gamma_{i}+n_{\mathrm{er}} \cos \gamma_{i}^{\mathrm{t}}}}
\end{array}\right.
$$

are the reflection coefficients facilitating the computation of energy of the reflected evanescent wave at the $i$-th facet, i.e. the share of energy that is not involved in the 
formation of the components of the optical force, and determination of the part of the energy of the refracted wave resulting in spatial motion of the RBC.

For the refracted evanescent wave, the components of the complex amplitude are written as:

$$
\begin{aligned}
& {\left[E_{\mathrm{ev} x}^{\mathrm{t}}\right]_{i}=\left[t_{\|}\right]_{i}\left[E_{\mathrm{ev} \|}\right]_{i} \sin \gamma_{i}^{\mathrm{t}} \sin \varphi_{i}} \\
& {\left[E_{\mathrm{ev} y}^{\mathrm{t}}\right]_{i}=\left[t_{\perp}\right]_{i}\left[E_{\mathrm{ev} \perp}\right]_{i} \cos \varphi_{i} \sin \delta_{i}} \\
& {\left[E_{\mathrm{ev} z}^{\mathrm{t}}\right]_{i}=-\left[t_{\|}\right]_{i}\left[E_{\mathrm{ev} \|}\right]_{i} \cos \gamma_{i}^{\mathrm{t}} \cos \varphi_{i} \cos \delta_{i}}
\end{aligned}
$$

Here

$$
\left\{\begin{array}{l}
{\left[t_{\| l}\right]_{i}=\frac{2 n_{\mathrm{sol}} \cos \gamma_{i}}{n_{\mathrm{er}} \cos \gamma_{i}+n_{\mathrm{sol}} \cos \gamma_{i}^{\mathrm{t}}}} \\
{\left[t_{\perp}\right]_{i}=\frac{2 n_{\mathrm{sol}} \cos \gamma_{i}}{n_{\mathrm{er}} \cos \gamma_{i}^{\mathrm{t}}+n_{\mathrm{sol}} \cos \gamma_{i}}}
\end{array}\right.
$$

are the refraction coefficients for the parallel and perpendicular components of an evanescent wave that impinges onto the $i$-th facet of an erythrocyte. $\left[E_{\mathrm{ev} \|}\right]_{i}\left(\left[E_{\mathrm{ev} \perp}\right]_{i}\right)$ are the components of the electrical vector of the incident evanescent wave at the plane of incidence, at the $i$-th facet. Hence,

$$
\begin{aligned}
& {\left[E_{\mathrm{ev} \|}\right]_{i}=E_{\mathrm{ev}} \cdot\left(\cos \varphi_{i} \sin ^{2} \delta_{i} \sin \gamma_{i}+\cos \varphi_{i} \cos ^{2} \delta_{i} \sin \gamma_{i}-\sin ^{2} \varphi_{i} \cos \gamma_{i}\right)} \\
& {\left[E_{\mathrm{ev} \perp}\right]_{i}=E_{\mathrm{ev}} \cdot\left(\cos \varphi_{i} \sin ^{2} \delta_{i} \cos \alpha_{i}+\cos \varphi_{i} \cos ^{2} \delta_{i} \sin \alpha_{i}+\sin ^{2} \varphi_{i} \sin \alpha_{i}\right)}
\end{aligned}
$$

are the fields for the $i$-th facet.

Determination of the optical force in the YOZ-plane with the components in the longitudinal and transversal directions (being typical for an evanescent wave) for the azimuth of polarization of the incident wave $\pm 45^{\circ}$ requires computation of the density of the spin and orbital angular momenta at the directions $O Y$ and $O Z$. The components are initially computed for each facet, and then the corresponding components are summed. The magnitudes of the components of the refracted evanescent wave at each facet are used for obtaining the estimation of the spin orbital momentum density and the orbital angular momentum density in the longitudinal and transversal directions.

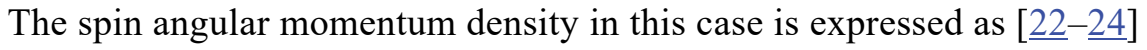

$$
\left[p_{\mathrm{s}}\right]_{i}=\frac{1}{16 \pi \omega} \nabla \times \operatorname{Im}\left[\left[\mathbf{E}_{\mathrm{ev}}^{\mathrm{t}^{*}}\right]_{i} \times\left[\mathbf{E}_{\mathrm{ev}}^{\mathrm{t}}\right]_{i}\right]
$$

Here $\left[\mathbf{E}_{\mathrm{ev}}^{\mathrm{t}}\right]_{i}$ is the transmitted evanescent wave interacting with the $i$-th facet of the erythrocyte. This expression contains both the longitudinal and transversal components [17]. The orbital angular momentum is given by 


$$
\left[p_{\mathrm{o}}\right]_{i}=\frac{1}{8 \pi \omega} \operatorname{Im}\left[\left[\mathbf{E}_{\mathrm{ev}}^{t *}\right]_{i} \cdot(\nabla)\left[\mathbf{E}_{\mathrm{ev}}^{t}\right]_{i}\right]
$$

The orbital (canonical) angular momentum caused by light pressure determines the force transferred to a cell in the direction of propagation of an evanescent wave.

Thus, the resulting momentum density in the $O Z$-direction is given by

$$
\begin{aligned}
{\left[p_{z}\right]_{i} } & =\left[p_{\mathrm{o}_{z}}\right]_{i}+\left[p_{\mathrm{s}_{z}}\right]_{i} \\
& =\frac{\left[t_{\|}\right]_{i}^{2} \cos ^{2}\left(\gamma_{i}^{\mathrm{t}}\right) \cos \left(\delta_{i}^{2}\right) A^{2}}{8 \pi \omega}\left[\left(k_{z}+\frac{m^{2} k^{2}}{k_{z}}+\frac{\kappa^{2}}{k_{z}}\right)-2 \frac{\kappa^{2}}{k_{z}}\right] \exp (-2 \kappa x)
\end{aligned}
$$

and the transversal momentum caused by the vertical spin becomes

$$
\left[p_{y}\right]_{i}=\left[p_{\mathrm{s}_{y}}\right]_{i}=\frac{\left[t_{\perp}\right]_{i}^{2} \sin ^{2}\left(\delta_{i}^{2}\right) A^{2}}{4 \pi \omega} \frac{k \kappa}{k_{z}} \operatorname{Im}(m) \exp (-2 \kappa x)
$$

where $A=E \frac{\cos \varphi_{i}}{\sqrt{1+|m|^{2}}}$.

Let us compute the optical force $\mathbf{F}_{i}$ acting on each facet of the RBC. We use the Mie light-scattering theory, under which approximation [24-26] $\mathbf{F}_{i}=\Delta \mathbf{p}_{i} S_{i}$, where $\Delta \mathbf{p}_{i}$ is the change of momentum density at the corresponding facet $S_{i}$ interacting with the evanescent wave for $i=1$ to $N$, where $N$ is the number of facets. The obtained quantities $\Delta\left[p_{y}\right]_{i}$ and $\Delta\left[p_{z}\right]_{i}$ give the possibility to calculate the components of the optical force $\left[F_{Y}\right]_{i}$ and $\left[F_{Z}\right]_{i}$.

The refracted evanescent wave in the $O X$-direction for each facet $i$ also forms some components of the optical force in this direction, which can be computed using the following relation:

$$
\left[F_{x}\right]_{i}=\iint_{\sigma}\left\langle\left[\mathbf{T}_{x}\right]_{i}\right\rangle \hat{n} \mathrm{~d} S_{i}
$$

where $\left[\mathbf{T}_{x}\right]_{i}$ is the component of the time-averaged Maxwell's stress-vector

$$
\left\langle\mathbf{T}_{i}\right\rangle=\frac{\varepsilon_{0}}{2}\left[\left[\mathbf{E}_{\mathrm{ev}}^{\mathrm{t}}\right]_{i}\left[\mathbf{E}_{\mathrm{ev}}^{\mathrm{t}}\right]_{i}^{*}+c^{2}\left[\mathbf{B}_{\mathrm{ev}}^{\mathrm{t}}\right]_{i}\left[\mathbf{B}_{\mathrm{ev}}^{\mathrm{t}}\right]_{i}^{*}-\frac{1}{2}\left(\left|\left[\mathbf{E}_{\mathrm{ev}}^{\mathrm{t}}\right]_{i}\right|^{2}+c^{2}\left|\left[\mathbf{B}_{\mathrm{ev}}^{\mathrm{t}}\right]_{i}\right|^{2}\right) I\right]
$$

computed for the $i$-th facet in the $O X$-direction. Here $I$ is the unit matrix, $\hat{n}$ is the surface-normal vector, $\mathbf{B}_{\mathrm{ev}}^{\mathrm{t}}=\sqrt{\varepsilon \varepsilon_{0}} \mathbf{E}_{\mathrm{ev}}^{\mathrm{t}}$ is the magnetic inductance vector for the evanescent wave, and $\varepsilon$ is the media permittivity.

The refracted evanescent wave (inhomogeneous in intensity) caused by the action of the gradient force

$$
\left[\mathbf{F}_{\text {grad }}\right]_{i}=-\frac{1}{2} n \nabla\left[\mathbf{E}_{\mathrm{ev}}^{\mathrm{t}}\right]_{i}^{2}
$$


is connected with the direction of propagation of the beam refracted at the $i$-th facet of the beam. The total component of the optical force in the $O X$-direction for the $i$-th facet is $\left[F_{X}\right]_{i}=\left[F_{x}\right]_{i}+\left[F_{\operatorname{grad} x}\right]_{i}$. The next calculation step consists in recalculation of the found set of optical force components for each of the specified facets $\left[F_{X}\right]_{i},\left[F_{Y}\right]_{i}$, and $\left[F_{Z}\right]_{i}$ into the resulting components, by summation of the corresponding force components over all facets of the erythrocyte surface

$$
F_{m}=\sum_{i}^{N}\left[F_{m}\right]_{i}, \quad m=X, Y, Z
$$

The computed results show that the magnitude of the components of the force $F_{X}$ is not large enough to provide the vertical motion of the $\mathrm{RBC}$, which is by several orders of magnitude smaller than the magnitude of the longitudinal component, and therefore has no influence on the spatial motion of a cell. The estimated ratio of the components of the optical force in the longitudinal and transversal directions also shows that the longitudinal force's component (in the OZ-direction) is much larger than the transversal component. Thus, rectilinear motion of investigated erythrocyte will be observed in the longitudinal $O Z$-direction. For visualization of such a motion of a cell in the $O Y$-direction, illumination of an erythrocyte by one wave is not a sufficient condition, forcing the search for alternative approaches for demonstration of such motions. The action of an evanescent wave on erythrocyte in this model experiment also manifests itself in an observed rotational motion of a cell with respect to its axis of symmetry.

\subsection{The computation of the optical force generated by two opposite propagated evanescent waves}

In accordance with the above represented scheme for computation of the optical force generated by the evanescent wave, one determines the force components $F_{m_{1}}$ and $F_{m_{2}}$ for the first (I) and second (II) (Fig. 1) evanescent wave acting on the erythrocyte. As the preliminary simulation shows, the incidence of the beams onto the surface of a prism - where total internal reflection appears - must be symmetrical with respect to the axis of symmetry of an erythrocyte $O O^{\prime}$.

Let the polarizations of the two beams be the same, i.e. the azimuths of polarization of both beams are equal to $+45^{\circ}$ or $-45^{\circ}$. Thus, for the symmetrical incidence of the beams, both the total longitudinal component of the optical force $F_{Z}=F_{Z_{1}}+F_{Z_{2}}$, and the total transversal component $F_{Y}=F_{Y_{1}}+F_{Y_{2}}$, become equal zero. $F_{Y_{1}, Y_{2}}$ and $F_{Z_{1}, Z_{2}}$ are the transversal and longitudinal components, respectively, of the evanescent waves excited by the linearly polarized incident waves with azimuths of polarization $\pm 45^{\circ}$. Evanescent waves formed above the prism surface are elliptically polarized, with the same direction of rotation of the electric-field vector, each of which has a torque, which is inherent in the vertical spin of an evanescent wave. Such action results in the total torque that is of twice as large for the chosen angles.

Formation of the transversal component of the optical force and demonstration of its directional action presumes the use of two linearly polarized incident beams whose 
azimuths of polarization are orthogonal, viz. $+45^{\circ}$ and $-45^{\circ}$ ( $c f$. Fig. 1). Elliptically polarized evanescent waves with the opposite directions of rotation of the electric field vectors compensate the resulting rotational motion of a cell and provide the condition for observation of a rectilinear motion of the RBC. In accordance with the conditions of modelling, for compensated longitudinal component $F_{Z}=F_{Z_{1}}+F_{Z_{2}}$, one observes a strengthening of the transversal component, which can be visualized experimentally. The magnitude of the transversal component and its influence on the characteristics of motion of a cell depend on the angle of incidence of waves on the erythrocyte surface and determine the velocity of a cell's spatial motion. In our case $F_{Y}=F_{Y_{1}}+F_{Y_{2}}$. This enables variation of the spatial position of an erythrocyte. Let us assume that the transversal forces formed because of the action of an evanescent wave do not cause deformation of a moving erythrocyte. The parameters of erythrocyte shape used in simulation have been obtained from the experimental data [20]. The change of the incidence angle of the beam on the surface of the prism changes the value of the transverse component of the optical force, thereby making it possible to control the spatial position of the erythrocyte. The maximal magnitude of the resulting optical force occurs for an angle of incidence of $63^{\circ}$.

Transversal motion of an erythrocyte in blood plasma is caused by the action of the resulting force $\mathbf{F}=\mathbf{F}_{\text {opt }}+\mathbf{F}_{\text {st }}$, where $\mathbf{F}_{\text {opt }}=\mathbf{F}_{Y}$ is the optical force. Since optical power is independent of time, it became possible to write these equations. A preliminary assessment of the gravity and buoyant forces showed that their values are several orders of magnitude smaller than the optical power or the Stokes force; therefore, these forces were not taken into account while estimating of the velocity of the red blood cell.

At low Reynolds numbers, the damping factor or drag coefficient is estimated by approximating the disk shape of an erythrocyte by a sphere of the same cross-sectional area, then $F_{\text {st }}=3 \pi \eta D v$ is the Stokes force. Here $\eta=1.236 \times 10^{-3} \mathrm{~Pa} \cdot \mathrm{s}$ is the dynamic

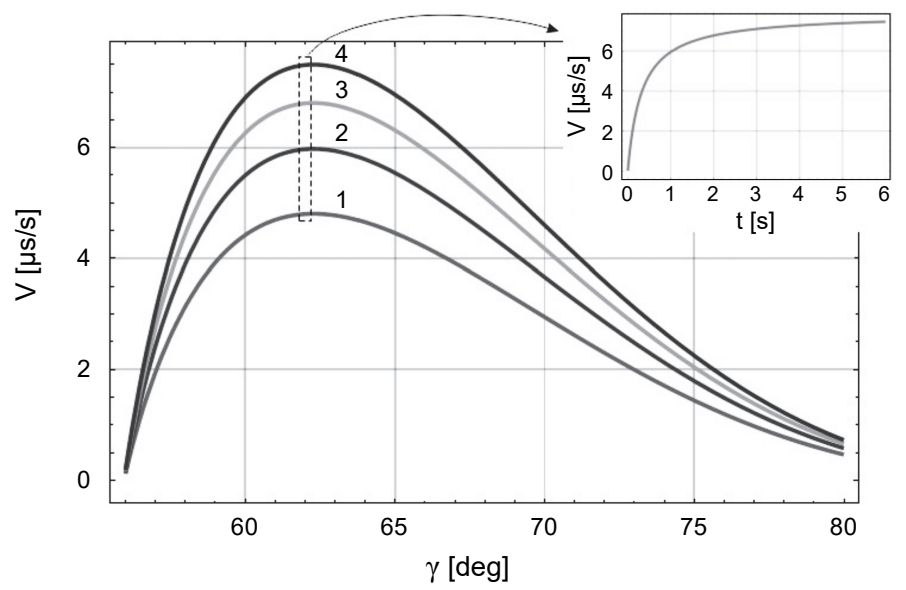

Fig. 3. Velocity of an erythrocyte in the transversal direction depending on the angle of illumination for different moments of time: $0.5 \mathrm{~s}$ (curve 1), $1 \mathrm{~s}$ (curve 2), $2 \mathrm{~s} \mathrm{(curve} \mathrm{3),} \mathrm{and} 6 \mathrm{~s}$ (curve 4); the inset indicates the velocity change with time for the angle of incidence of $62^{\circ}$. 
viscosity of blood plasma, $D$ is the diameter of the corresponding cross-sectional area of the cell, and $\mathrm{v}$ is the velocity of the RBC. While

$$
m \frac{\mathrm{d} v}{\mathrm{~d} t}=F_{Y}+3 \pi \eta D v
$$

one can determine the velocity of erythrocyte in a solution at the transversal direction as a function of the angle of incidence (Fig. 3), by comparing the theoretical and experimental results. The change in the irradiation conditions leads to a significant change in the speed of motion of an erythrocyte. Figure 3 demonstrates the evolution of the velocity of erythrocyte motion at fixed times of the action of the evanescent wave on it.

In the course of time, the velocity of an erythrocyte is experimentally detected while changing the angle of illumination.

\section{Experimental demonstration of transversal motion of erythrocytes into evanescent field}

The results of computer simulation of moving erythrocytes in an evanescent field will now be supported experimentally. A blood plasma is prepared by centrifugation of fresh venous blood at room temperature. Initially, a whole blood $(1500 \mathrm{~g})$ is centrifuged during $10 \mathrm{~min}$, which results in separation of sedimented erythrocytic masses from plasma. Further, a second centrifugation ( $1500 \mathrm{~g}$, here $g=9.8 \mathrm{~m} / \mathrm{s}^{2}$ ) during $10 \mathrm{~min}$ is carried out at room temperature for removing residual cells, such as thrombocytes. As an anticoagulant, ethylenediaminetetraacetic acid was used. Then, blood from a finger $(0.48 \mu \mathrm{l})$ is diluted in $1 \mathrm{ml}$ of blood plasma. The prepared suspension $(50 \mu \mathrm{l})$ is placed in a hermetic cuvette consisting of a cover glass of $0.15 \mathrm{~mm}$ thickness and the total reflection prism at a distance $0.05 \mathrm{~mm}$. To avoid adhesion of erythrocytes, a cover glass and the prism surface are sequentially washed out in a solution of surface-active material, in $96 \%$ ethyl alcohol, then in deionized water, and finally in $3 \%$ solution of hydrogen peroxide, and then dried and washed out in $2 \%$ solution of human albumin. The elements of the blood that interfered were removed from the field of view with the help of laser tweezers (gradient traps).

The optical arrangement for experimental demonstration of erythrocyte motion in an evanescent field is shown in Fig. 4.

The radiation from a semiconductor laser 1 with wavelength $\lambda=980 \mathrm{~nm}$ and power $1 \mathrm{~W}$ passes the beam expander 2 consisting of two objectives and a micron-scale pinhole as the spatial frequency filter. We varied the radiation power from 5 to $500 \mathrm{~mW}$. The beam with a plane wavefront passes a quarter-wave plate 3 and becomes circularly polarized. After beam splitter 4, two beams of equal intensities are reflected by the mirrors 5 to the total reflection prism 7. Polarizers 6 form the orthogonally linearly polarized beams with the azimuths of polarization $\pm 45^{\circ}$, which fall on the interface glass-blood plasma. In a cuvette 8 with erythrocytes, two oppositely directed evanescent waves of equal intensities are formed. Moving RBCs are observed in white light from a LED display 9 


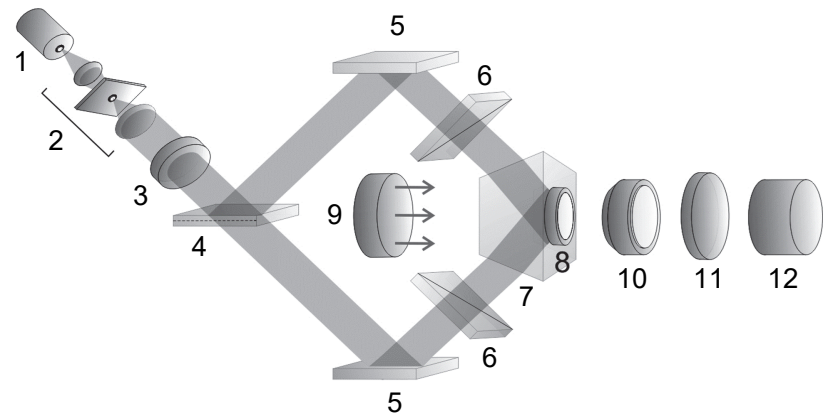

Fig. 4. Optical arrangement for study of moving erythrocytes in an evanescent field: 1 - laser, 2 - beam expander with a spatial filter, 3 - quarter-wave plate, 4 - beam splitter, 5 - mirrors, 6 - polarizers, 7 total reflection prism, 8 - position of erythrocytes, 9 - LED display, 10 - microobjective, 11 - light filter, and $12-$ CCD camera.

using a microobjective 10 (magnification 60×) and CCD camera 12. Light filter 11 cuts off radiation from the IR laser.

Taking into consideration the results of the theoretical simulation, the corresponding experimental conditions were chosen, namely, the incidence angle of opposite beams at the interface of the "prism-blood plasma", maintaining the azimuth of polarization $\pm 45^{\circ}$ of these beams.

Motion of an erythrocyte in opposite evanescent waves is illustrated in Fig. 5.
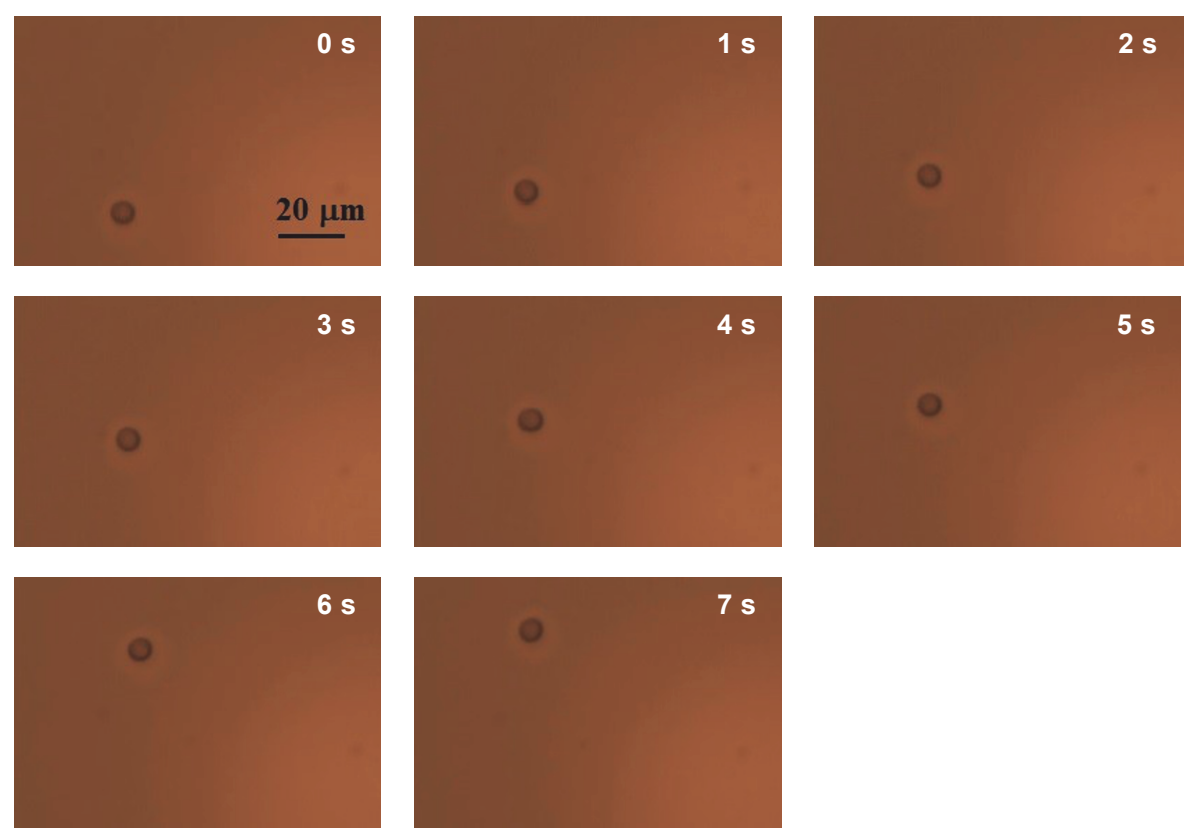

Fig. 5. Motion of an erythrocyte in opposite evanescent waves. The frame frequency is $1 \mathrm{~Hz}$. 
The frame frequency equals $1 \mathrm{~Hz}$. One observes only transversal motion of an erythrocyte with a speed $6 \mu \mathrm{m} / \mathrm{s}$, approximately. The longitudinal moving is compensated by the opposite beams, while the transversal moving for linearly polarized beams with the azimuth of polarization $+45^{\circ}$ doubles, in agreement with the theoretical prediction. One observes correlation of the results of computer simulation and the experimental study with a discrepancy not exceeding $30 \%$.

\section{Conclusion}

A theoretical model for calculating the transverse motion of the erythrocyte in the evanescent field formed by two opposite evanescent waves is suggested. The obtained field is the result of total internal reflection at the "prism-blood plasma" interface of incident linearly polarized waves with the azimuth of polarization of $\pm 45^{\circ}$. The generated evanescent field penetrates into the erythrocyte, causing its motion. The above experimental results confirm the possibility of transversely controlled motion of a microobject due to the influence of the vertical spin of evanescent waves. Specifically, the discrepancy between the theoretical and experimental results does not exceed $30 \%$.

\section{References}

[1] XIN H., Li B., Targeted delivery and controllable release of nanoparticles using a defect-decorated optical nanofiber, Optics Express 19(14), 2011, pp. 13285-13290, DOI: 10.1364/OE.19.013285.

[2] Dholakia K., Reece P., Optical micromanipulation takes hold, Nano Today 1(1), 2006, pp. 18-27, DOI: $10.1016 / \mathrm{S} 1748-0132(06) 70019-6$.

[3] Wang M. D., Yin H., Landick R., Gelles J., Block S.M., Stretching DNA with optical tweezers, Biophysical Journal 72(3), 1997, pp. 1335-1346, DOI: 10.1016/S0006-3495(97)78780-0.

[4] Bustamante C., Bryant Z., Smith S.B., Ten years of tension: single-molecule DNA mechanics, Nature 421(6921), 2003, pp. 423-427, DOI: 10.1038/nature01405.

[5] Altman D., Sweeney H.L., Spudich J.A., The mechanism of myosin VI translocation and its load -induced anchoring, Cell 116(5), 2004, pp. 737-749, DOI: 10.1016/S0092-8674(04)00211-9.

[6] Asbury C.L., Fehr A.N., Block S.M., Kinesin moves by an asymmetric hand-over-hand mechanism, Science 302(5653), 2003, pp. 2130-2134, DOI: 10.1126/science.1092985.

[7] Zenkova C.Yu., Gorsky M.P., Ryabiy P.A, Gruia I., Different approaches to phase restoration of distant complex optical fields, Optica Applicata 45(2), 2015, pp. 139-150, DOI: 10.5277/oa150201.

[8] Zenkova C.Yu., GoRsky M.P, Ryaby P.A., The phase problem solving by the use of optical correlation algorithm for reconstructing phase skeleton of complex optical fields, Proceedings of SPIE $\mathbf{9 2 5 8}$, 2015, article ID 92582B, DOI: 10.1117/12.2070415.

[9] Zenkova C.Yu., Gorsky M.P., Ryabiy P.A., Angelskaya A.O., Additional approaches to solving the phase problem in optics, Applied Optics 55(12), 2016, pp. B78-B84, DOI: 10.1364/AO.55.000B78.

[10] Zenkova C.Yu., GoRsky M.P., RYABIY P.A., Pseudo-phase mapping of speckle fields using 2D Hilbert transformation, Optica Applicata 46(1), 2016, pp. 153-162, DOI: 10.5277/oa160114.

[11] Zenkova C.Yu., Gorsky M.P., Soltys I.V., Angelsky P.O., Use of motion peculiarities of test particles for estimating degree of coherence of optical fields, Ukrainian Journal of Physical Optics 13(4), 2012, pp. 183-195.

[12] ZenKova C.Yu., Interconnection of polarization properties and coherence of optical fields, Applied Optics 53(10), 2014, pp. B43-B52, DOI: 10.1364/AO.53.000B43. 
[13] Zenkova C., Soltys I., Angelsky P., The use of motion peculiarities of particles of the Rayleigh light scattering mechanism for defining the coherence properties of optical fields, Optica Applicata 43(2) 2013, pp. 297-312, DOI: 10.5277/oa130210.

[14] Byrne G.D., Pitter M.C., Zhang J., Falcone F.H., Stolnik S., Somekh M.G., Total internal reflection microscopy for live imaging of cellular uptake of sub-micron non-fluorescent particles, Journal of Microscopy 231(1), 2008, pp. 168-179, DOI: 10.1111/j.1365-2818.2008.02027.x.

[15] Tuchin V., Optical Biomedical Diagnostics, Vol. 1, 2017 (in Russian).

[16] Kugeyro M.M., Smunyov D.A., Determination of microphysical parameters of native erythrocytes on the results of measuring the optical characteristics of scattered radiation, Bulletin of Belarusian State University 1(2), 2016, p. 73 (in Russian).

[17] YuRKin M.A., Modelling of light scattering by blood cells using the discrete dipoles method, $\mathrm{PhD}$ Thesis, Novosibirsk, 2008 (in Russian).

[18] Ushenko A.G., Ermolenko S.B., Burkovets D.N., Ushenko Y.A., Polarization microstructure of laser radiation scattered by optically active biotissues, Optics and Spectroscopy 87, 1999, pp. 434-438.

[19] Angelsky O.V., Ushenko Y.A., Dubolazov A.V., Telenha O.Yu., The interconnection between the coordinate distribution of Mueller-matrixes images characteristic values of biological liquid crystals net and the pathological changes of human tissues, Advances in Optical Technologies, Vol. 2010, 2010, article ID 130659, DOI: 10.1155/2010/130659.

[20] Gu M., Kuriakose S., GAN X., A single beam near-field laser trap for optical stretching, folding and rotation of erythrocytes, Optics Express 15(3), 2007, pp. 1369-1375, DOI: 10.1364/OE.15.001369.

[21] Hayat A., Balthasar Mueller J.P., CAPAsso F., Lateral chirality-sorting optical forces, PNAS Early Edition 112(43), 2015, pp. 13190-13194, DOI: 10.1073/pnas.1516704112.

[22] Angelsky O.V., Hanson S.G., Maksimyak P.P., Maksimyak A.P., Zenkova C.Yu., Polyanskil P.V., IVANSKYI D.I., Influence of evanescent wave on birefringent microplates, Optics Express 25(3), 2017, pp. 2299-2311, DOI: 10.1364/OE.25.002299.

[23] Bliokn K.Y., Bekshaev A.Y., Nori F., Dual electromagnetism: helicity, spin, momentum, and angular momentum, New Journal of Physics 15(3), 2013, article ID 033026, DOI: 10.1088/1367-2630/15/3/ $\underline{033026 .}$.

[24] Antognozzi M., Bermingham C.R., Harniman R.L., Simpson S., Senior J., Hoerber H., Dennis M.R., Bekshaev A.YA., Bliokh K. Y., Nori F., Direct measurements of the extraordinary optical momentum and transverse spin-dependent force using a nano-cantilever, Nature Physics 12, 2016, pp. 731-735, DOI: $10.1038 /$ nphys3732.

[25] Zenkova C.Yu., Ivanskyi D.I., Kiyashchuk T.V., Optical torques and forces in birefringent microplate, Optica Applicata 47(3), 2017, pp. 483-493, DOI: 10.5277/oa170313.

[26] ZENKOVA C.YU., IVANSKYI D.I., Non-trivial structure of optical momentum and optical forces inherent in evanescent waves, Proceedings of SPIE 10612, 2018, article ID 1061207, DOI: 10.1117/12.2303555.

[27] Bekshaev A.Ya., Angelsky O.V., Sviridova S.V., Zenkova C.Yu., Mechanical action of inhomogeneously polarized optical fields and detection of the internal energy flows, Advances in Optical Technologies, Vol. 2011, 2011, article ID 723901, DOI: 10.1155/2011/723901. 